\title{
On the role of global flow instability analysis in closed loop flow control
}

\author{
F. Gómez*, V. Theofilis, P. Paredes, Q. Liu, W. He \\ School of Aerospace Engineering, Universidad Politécnica de Madrid, Spain
}

\begin{abstract}
Control of linear flow instabilities has been demonstrated to be an effective theoretical flow control methodology, capable of modifying transitional flows on canonical geometries such as the plane channel and the flat-plate boundary layer. Extending the well-developed theoretical flow control techniques to flows over or through complex geometries requires addressing the issue of efficient capturing of the leading members of the global eigenspectrum pertinent to such flows. The present contribution describes state-of-the-art modal global instability analysis methodologies recently developed in our group, based on matrix formation and time-stepping, respectively. The relative performance of these algorithms is assessed on the recovery of BiGlobal and TriGlobal eigenspectra in the spanwise periodic and the cubic lid-driven cavity, respectively; the adjoint eigenspectrum in the latter flow is recovered for the first time. For three-dimensional flows without any homogeneous spatial direction, the time-stepping methodology was found to outperform the matrix-forming approach and permit recovering the leading TriGlobal eigenmodes in an three-dimensional open cavity of aspect ratio $L: D: W=5: 1: 1$; theoretical flow control of this configuration is underway.
\end{abstract}

\section{Introduction}

Theoretical active flow control, via control of linear flow instabilities, can alter the flow state in a desired manner through either attenuation or amplification of intrinsic small-amplitude flow perturbations, at the expense of relatively small amounts of actuator power compared with that necessary to control the nonlinear flow. Ever expanding flow control concepts and improving technologies for the construction of actuators capable of controlling a flow at ever smaller spatial scales, ${ }^{1}$ combined with continuous progress in the identification and understanding of fluid flow instabilities, increasingly convert flow control from art to science, in which the input-output dynamic behavior is described by an appropriately defined state-space formulation. ${ }^{2}$ Successes have been reported in the literature over the past two decades, initially focusing on regular geometries in which canonical flows, having one inhomogeneous and either two homogeneous (e.g. plane channel) or one homogeneous and one weakly-developing (e.g. flat-plate boundary layer) spatial direction were considered ${ }^{3,4}$

However, most interesting flows targeted by flow control develop over or through complex geometries in which all three spatial directions are inhomogeneous. In certain limiting cases parts of the field may be identified in which the flow can be taken to depend in an inhomogeneous manner upon two spatial directions and either be homogeneous or develop weakly along the third spatial direction. Hydrodynamic and aeroacoustic small-amplitude perturbations in both of these classes of flows are unraveled by global linear theory. ${ }^{5}$ Though conceptually straightforward, the extension of the theoretical flow control ideas demonstrated in the classic flat plate and cylinder geometries ${ }^{2,3}$ into flows developing in three-dimensional geometries without any homogeneous spatial direction stumbles upon the issues of efficiency and accuracy of the numerical work underlying recovery of the global eigenspectrum, the latter being inherent to the operator discretizing the linearized Navier-Stokes equations. The relation of this operator to flow control is two-fold: when flow control focuses on control of flow instabilities, the same operator is at the core of the linear state space formulation. When flow control is guided by the identification of spatial regions of high flow sensitivity, both the discretized linearized Navier-Stokes operator and its adjoint need be computed. ${ }^{6,7}$

*also at Instituto Nacional de Técnica Aeroespacial 
In the last decade flow control via control of global flow instabilities has also been reported in the literature, ${ }^{8}$ expedited by Galerkin projection of the full system onto an appropriately defined low-dimensional basis, a Reduced Order Model (ROM) designed to describe the system dynamics. ${ }^{9,10}$ From a practical point of view, use of a ROM circumvents working with the full system matrix, which offers the key advantage of a drastic reduction in the number of degrees of freedom compared with that corresponding to the discretized linear state-space formulation which describes feedback control of the flow in question; in this manner, controllers of manageable size may be constructed. An obvious drawback of embedding a ROM in the overall control algorithm is the a-priori unknown performance (or even validity) of the proposed ROM when flow conditions are changed.

Last but not least, the theoretically unresolved issue of flow turbulence also affects theoretical flow control. Although evidence exists that linear theory does aid prediction of turbulent shear flows, ${ }^{11}$ the authors are unaware of any effort which address the issue of control of turbulent flows in three-dimensional geometries without homogeneous spatial directions via control of (global) instabilities of turbulent flows. From a numerical point of view, again, the formidable size of the matrix discretizing the spatial operator of the linearized equations of motion is a show-stopper hampering progress in this direction. Quoting from the Future Issues section of a recent reference on global instability, ${ }^{5}$

2 Algorithms that circumvent matrix storage and inversion hold promise to provide the breakthrough necessary for TriGlobal flow instability analysis (and control).

$\cdots$

4 Treatment of turbulence in predictive instability analysis and control models needs to be addressed, focusing on complex geometries.

The present contribution is concerned with addressing the first of these major open issues, namely efficient recovery of the global eigenspectrum in fully three-dimensional flows, i.e. those developing in domains without any homogeneous spatial directions. A matrix-formation method is employed for the first time to the recovery of the three-dimensional adjoint eigenspectrum in such flows. In addition, a recently devised accurate and flexible time-stepping method ${ }^{12}$ is employed to unravel the direct eigenspectrum in arbitrarily complex three-dimensional geometries, by coupling the time-stepping algorithm with the open source CFD software OpenFOAM ${ }^{\circledR}$. The theoretical background is briefly summarized in $\S$ II.A. Both algorithms for the recovery of the eigenspectrum are discussed in $\S$ III, where it is shown that three-dimensional (TriGlobal) instability analysis work (and the associated flow control) at low Reynolds numbers may be performed on standard desktop computers. Results on the direct and adjoint eigenspectra in the cubic lid-driven cavity, obtained by both algorithms, are shown in $\S I V$, where the limits of the matrix-forming methodology when solving the problem serially are identified. Attention is finally focused only on the time-stepping methodology using which the leading members of the eigenspectrum of an aspect-ratio 5:1:1 open cavity are obtained for the first time. Brief concluding remarks are offered in $\S \mathrm{V}$.

\section{Theoretical considerations}

\section{II.A. The spatial discretization matrix A in (closed-loop) flow control}

The linear state space system describing input-output small-amplitude perturbation dynamics can be written as

$$
\begin{aligned}
& \dot{\hat{\mathbf{q}}}=\mathbf{A} \hat{\mathbf{q}}+\mathbf{B u} \\
& \mathbf{y}=\mathbf{C} \hat{\mathbf{q}}+\mathbf{D u}
\end{aligned}
$$

where $\hat{\mathbf{q}} \in \mathbb{R}^{n}$ is the vector of linear flow perturbations, $\mathbf{A} \in \mathbb{R}^{n \times n}$ is the operator describing spatial discretization of the linearized Navier-Stokes equations (LNSE), linearization being performed around the base state $\overline{\mathbf{q}}$ which is to be controlled, $\mathbf{u} \in \mathbb{R}^{m}$ and $\mathbf{y} \in \mathbb{R}^{r}$ are the vectors of inputs and outputs, respectively, while the input matrix $\mathbf{B} \in \mathbb{R}^{n \times m}$ describes the actuators, and the output matrix $\mathbf{C} \in \mathbb{R}^{r \times n}$ describes the sensors. The feed-through matrix $\mathbf{D} \in \mathbb{R}^{r \times m}$ is taken to be non-zero when $\mathbf{u}$ directly affects $\mathbf{y}$, otherwise it is neglected. The usual assumption $m, r \ll n$ implies that the dimension of the discretized system (1-2) is dominated by $n$, the number of degrees of freedom which discretize the LNSE. In turn, $n$ is determined 
by whether the flow to be controlled is incompressible or compressible and also whether it is developing in a domain with one, two or three inhomogeneous spatial directions.

A Laplace transformation of the system (1-2) leads to the definition of the transfer function, $\mathbf{G}(s) \in \mathbb{C}^{r \times m}$, through which the frequency response matrix, $\mathbf{G}(\mathrm{i} \omega)$, may also be defined,

$$
\mathbf{G}(s)=\mathbf{C}(s \mathbf{I}-\mathbf{A})^{-1} \mathbf{B}+\mathbf{D}, \quad \mathbf{G}(\mathrm{i} \omega)=\mathbf{C}(\mathrm{i} \omega \mathbf{I}-\mathbf{A})^{-1} \mathbf{B}+\mathbf{D},
$$

with $s$ the Laplace-transformed time-coordinate, $\omega$ a real frequency and $\mathbf{i}=\sqrt{-1}$.

From a purely formal point of view, computation of the frequency response matrix and recovery of a window of the global flow eigenspectrum after a complex shift, ${ }^{5}$ share the common need to calculate the inverse of matrix $(\mu \mathbf{I}-\mathbf{A}), \mu \in \mathbb{C}$, which is circumvented in practice by calculating its LU decomposition. One key question regards how to compute, efficiently and accurately, this inverse matrix. Two procedures exist, one in which the matrix is formed and LU-decomposed, in order for the decomposition to be used during the construction of the Krylov subspace, and another in which the action of the operator is computed, without ever forming the matrix. These procedures are referred to as matrix-forming and time-stepping approaches, respectively; ${ }^{5}$ they are briefly exposed in what follows.

\section{II.B. Stability Analysis}

The three-dimensional dimensionless Navier-Stokes equations of a viscous incompressible flow can be summarized as:

$$
\frac{\partial \mathbf{q}}{\partial t}=\mathbf{f}(\mathbf{q})
$$

where $\mathbf{q}$ contains the (divergence-free) three velocity components in the computational nodes.

Linear stability theory studies the temporal evolution of small amplitude disturbances superimposed upon a base flow. Linear TriGlobal instability analysis proceeds by decomposing a field in a base flow $\overline{\mathbf{q}}=[U, V, W, P]^{T}(x, y, z)$ and small-amplitude three-dimensional unsteady perturbations $\hat{\mathbf{q}}$. A linearized initial value problem can be written taking this assumption into the Navier-Stokes equation (4), retaining the infinitesimal terms:

$$
\frac{\partial \hat{\mathbf{q}}}{\partial t}=\frac{\partial \mathbf{f}(\overline{\mathbf{q}})}{\partial \mathbf{q}} \hat{\mathbf{q}} \equiv \hat{\mathbf{q}},
$$

where $\mathbf{A}$ is the Jacobian matrix of the Right Hand Side of the Navier-Stokes equation. According to the TriGlobal Ansatz, ${ }^{13}$ in a modal analysis context solutions of (5) are sought as eigenmodes:

$$
\hat{\mathbf{q}}(x, y, z, t)=\epsilon \hat{\mathbf{q}}^{\prime}(x, y, z) \mathrm{e}^{-\omega t}+c . c .
$$

where $\epsilon \ll 1, \omega=\omega_{r}+i \cdot \omega_{i}$, with $\omega_{i}$ representing a frequency and $\omega_{r}$ being the amplification/damping rate of the disturbance sought and c.c. the complex conjugate.

\section{II.C. Matrix formation for the three-dimensional direct and adjoint eigenvalue problem}

A three-dimensional flow, $\overline{\mathbf{q}}$, the linear instability and control of which are to be studied, admits smallamplitude modal perturbations having three-dimensional amplitude functions that are harmonic in time, of the form

$$
\hat{\mathbf{q}}=\hat{\mathbf{q}}^{\prime}(x, y, z) \exp [-\omega t], \quad \text { and } \quad \hat{\mathbf{q}}^{*}=\hat{\mathbf{q}}^{* \prime}(x, y, z) \exp \left[\omega^{*} t\right]
$$

for the direct and adjoint linear perturbations, respectively. Introduction of this class of disturbances into the direct and adjoint linearized Navier-Stokes and continuity equations, here taken incompressible and laminar for exposition purposes, results in the direct $\mathcal{A} \hat{\mathbf{q}}^{\prime}=\omega \mathcal{B} \hat{\mathbf{q}}^{\prime}$ and its corresponding adjoint $\mathcal{A}^{*} \hat{\mathbf{q}}^{* \prime}=\omega^{*} \mathcal{B}^{*} \hat{\mathbf{q}}^{* \prime}$ eigenvalue problem, where

$$
\begin{aligned}
& \mathcal{A}=\left(\begin{array}{cccc}
\mathcal{L}_{3 D}+U_{x} & U_{y} & U_{z} & \mathcal{D}_{x} \\
V_{x} & \mathcal{L}_{3 D}+V_{y} & V_{z} & \mathcal{D}_{y} \\
W_{x} & W_{y} & \mathcal{L}_{3 D}+W_{z} & \mathcal{D}_{z} \\
\mathcal{D}_{x} & \mathcal{D}_{y} & \mathcal{D}_{z} & 0
\end{array}\right), \quad \quad \mathcal{B}=\left(\begin{array}{cccc}
1 & 0 & 0 & 0 \\
0 & 1 & 0 & 0 \\
0 & 0 & 1 & 0 \\
0 & 0 & 0 & 0
\end{array}\right) \\
& \mathcal{A}^{*}=\left(\begin{array}{cccc}
\mathcal{L}_{3 D}^{*}+U_{x} & V_{x} & W_{x} & \mathcal{D}_{x} \\
U_{y} & \mathcal{L}_{3 D}^{*}+V_{y} & W_{y} & \mathcal{D}_{y} \\
U_{z} & V_{z} & \mathcal{L}_{3 D}^{*}+W_{z} & \mathcal{D}_{z} \\
\mathcal{D}_{x} & \mathcal{D}_{y} & \mathcal{D}_{z} & 0
\end{array}\right), \quad \mathcal{B}^{*}=\left(\begin{array}{cccc}
-1 & 0 & 0 & 0 \\
0 & -1 & 0 & 0 \\
0 & 0 & -1 & 0 \\
0 & 0 & 0 & 0
\end{array}\right),
\end{aligned}
$$


with the three-dimensional linear operators being $\mathcal{L}_{3 D}=U \mathcal{D}_{x}+V \mathcal{D}_{y}+W \mathcal{D}_{z}-\frac{1}{R e}\left(\mathcal{D}_{x x}+\mathcal{D}_{y y}+\mathcal{D}_{z z}\right)$ and $\mathcal{L}_{3 D}^{*}=-U \mathcal{D}_{x}-V \mathcal{D}_{y}-W \mathcal{D}_{z}-\frac{1}{R e}\left(\mathcal{D}_{x x}+\mathcal{D}_{y y}+\mathcal{D}_{z z}\right)$. Appropriate boundary conditions close the respective eigenvalue problems. The solution procedure involves the iterative generation of a Krylov subspace and application of the shift-and-invert Arnoldi algorithm. ${ }^{14}$ For the latter the LU decomposition of the matrix A, which discretizes the operators $\mathcal{A}$ or $\mathcal{A}^{*}$, is needed. For the three-dimensional eigenvalue problems (8-9) the LU-decomposition is the most expensive item of the entire numerical solution algorithm.

\section{II.D. The Chiba Time-Stepping algorithm combined with a generic DNS code}

By contrast to the modal approach, in a time-stepping (TS) approach no explicit modal dependence of the perturbations need be assumed. Instead, the initial value problem for the development in time of small-amplitude perturbations is solved. Stability analysis can be carried out once the base flow has been obtained. Chiba's approach ${ }^{15,16}$ is a Newton Krylov Jacobian-free method ${ }^{17}$ based on the temporal evolution of the perturbation superimposed upon the base flow. The Chiba TS algorithm follows the classic Arnoldi ${ }^{18}$ procedure, which consists of the generation of a Krylov subspace and its orthonormalization in order to create a reduced matrix that contains the leading eigenvalues of the original system. This methodology has two advantages: first, it obtains the Jacobian-vector products directly from an external simulation code (typically the Direct Numerical Simulation code used for the calculation of the base flow) by means of numerical differentiation using the concept of the Frèchet derivative as introduced by Keller, ${ }^{19}$

$$
\mathrm{e}^{\mathbf{A} \tau} \hat{\mathbf{q}} \approx \frac{\mathbf{f}(\overline{\mathbf{q}}+\epsilon \hat{\mathbf{q}}(\tau))-\mathbf{f}(\overline{\mathbf{q}})}{\epsilon}
$$

Second, at the same time, the algorithm applies an exponential transformation of the spectrum, which is a requirement in order to extract the leading eigenvalues from the Jacobian. The Krylov subspace itself is constructed by application of the code onto an initial vector; details may be found in Bagheri et al. ${ }^{20}$ and Theofilis ${ }^{5}$ for further discussion. The complete stability analysis algorithm can be summarized as follows:

S1: Set $t o l_{\text {Arnoldi }}$ and $N_{\text {Arnoldi }}$ (Arnoldi tolerance and maximum number of Arnoldi iterations)

S2: Set an initial condition $\hat{\mathbf{q}}_{\mathbf{1}}$

S3: Perform Arnoldi loop until convergence, $\left(l=1, \ldots, N_{\text {Arnoldi }}\right)$,

A1: Use the DNS to obtain $\mathbf{q}_{+}=\overline{\mathbf{q}}(\tau)+\epsilon \hat{\mathbf{q}}_{\mathbf{l}}(\tau)$ and $\mathbf{q}_{-}=\overline{\mathbf{q}}(\tau)-\epsilon \hat{\mathbf{q}}_{1}(\tau)$

A2: Apply an exponential transformation $e^{\mathbf{A} \tau} \hat{\mathbf{q}}_{\mathbf{l}}=\frac{\mathbf{q}_{+}-\mathbf{q}_{-}}{2 \epsilon}$

A3: Perform Gram-Schmidt orthonormalization $(i=1, \ldots, l)$

B1: Form a Hessenberg matrix $h_{i l}=\hat{\mathbf{q}}_{i}^{T} e^{\mathbf{A} \tau} \hat{\mathbf{q}}_{\mathbf{l}}$

B2: Orthogonalize $\hat{\mathbf{q}}_{\mathbf{l}+\mathbf{1}}=\mathrm{e}^{\mathbf{A} \tau} \hat{\mathbf{q}}_{\mathbf{l}}-\sum_{\mathbf{i}=\mathbf{1}}^{\mathbf{j}} \mathbf{h}_{\mathbf{i l}} \hat{\mathbf{q}}_{\mathbf{i}}$

B3: Normalize $h_{l+1, l}=\left\|\hat{\mathbf{q}}_{\mathbf{l}+\mathbf{1}}\right\|, \hat{\mathbf{q}}_{\mathbf{l}+\mathbf{1}}=\frac{\hat{\mathbf{q}}_{\mathbf{l}+\mathbf{1}}}{\mathbf{h}_{\mathbf{l}+\mathbf{1}, \mathbf{1}}}$

S4: Perform eigenvalue decomposition of the small matrix $\mathbf{H}$, which contains approximations of the leading eigenvalues of $e^{\mathbf{A} \tau}$

S5: Undo the exponential transformation.

Validation and verification results of this algorithm, originally proposed by Chiba ${ }^{15}$ and used for the first ever TriGlobal modal instability analysis by Tezuka and Suzuki, ${ }^{21}$ are found elsewhere. ${ }^{22}$ In the latter reference details are discussed on the complete implementation of this stability analysis method, including issues such as the control of the parameter $\epsilon$ or the selection of the initial condition $\hat{\mathbf{q}}_{\mathbf{1}}$. 


\section{The numerical work}

\section{III.A. Forming the matrix using FD-q spatial discretization}

Accuracy in the numerical solutions of (1-2) and the computation of (3) is of paramount significance, if reliable flow control results are to be obtained. When forming the matrix, accurate solution of the eigenvalue problems defined by (8-9) is implied. Extending common practice in fluid flow stability, spectral methods are usually employed for the spatial discretization, since they offer exponential decay of errors as the number of discretization nodes is increased. More significant for flows with multiple inhomogeneous directions is the ability of spectral methods to deliver, on a given affordable resolution, solutions with minimum error when compared with those produced by any other spatial discretization method.

Conventional wisdom is that the computational effort associated with matrix storage and inversion, which respectively scales as $O\left(n^{2}\right)$ and $O\left(n^{3}\right)$ as far as memory and CPU time for the LU decomposition of the matrix is concerned when dense linear algebra is used, is prohibitively large for global flow instability and control to be based on matrix formation techniques; consequently, time-stepping algorithms are used, in which the action of the linear operator is calculated without the matrices ever being formed. ${ }^{23,24}$ For the same reason of a large size of the discretized matrices describing the feedback control problem, which can hardly be managed in all but canonical base flows with one inhomogeneous spatial direction, a ROM of the flow dynamics is invariably constructed prior to applying flow control. ${ }^{25}$

Defying conventional wisdom, the present contribution focuses on the construction, storage and LUdecomposition of the discretized matrix describing linear state-space formulation, by exploiting the accuracy and efficiency properties of a novel stable high-order finite-difference spatial discretization scheme of $O(q)$, denoted as FD-q. ${ }^{26}$ These methods have been employed for the numerical solution of spatial BiGlobal, PSE-3D and TriGlobal linear stability problems on a typical desktop computer, ${ }^{27,28}$ all within a matrix formation, storage and inversion context.

The theoretical accuracy limit of the FD-q methods when $q \rightarrow N$, where $N$ is the number of nodes discretizing the $n$ degrees of freedom, is that of spectral collocation. Much more interesting from an efficiency point of view is the savings in both memory for the matrix storage, $\mathrm{Mem}_{F D-q}$, and CPU time for the LUdecomposition of the matrix, $\mathrm{T}_{F D-q}$, that the FD-q methods offer at a given accuracy, $O(q)$, when compared with spectral collocation based on $N$ Chebyshev Gauss-Lobatto (CGL) gridpoints. These savings have been quantified $^{28,29}$ as

$$
\operatorname{Mem}_{F D-q} \sim\left(\frac{q}{N}\right)^{2} \times \operatorname{Mem}_{C G L} \quad \text { and } \quad \mathrm{T}_{F D-q} \sim\left(\frac{q}{N}\right)^{3} \times \mathrm{T}_{C G L} .
$$

The question of optimal choice of $q$ has been addressed in detail by reference to the classic linear local, the BiGlobal or the TriGlobal LNSE operators to which (1-2) respectively reduces when $\mathbf{B}=\mathbf{C}=\mathbf{D}=0$ and flows with one, two or three inhomogeneous spatial directions are monitored. ${ }^{28,29}$

\section{III.B. The Chiba Time-Stepping algorithm combined with OpenFOAM ${ }^{\circledR}$}

The flexibility of the TS algorithm discussed earlier is exploited by coupling it to the open source computational mechanics software OpenFOAM ${ }^{\circledR}$ and using the latter for the DNS work to be described in what follows. Of all geometries analyzed Figure 1 (a) shows a structured mesh constructed using the OF blockMesh utility around a three-dimensional open cavity of aspect ratio $L: D: W=5: 1: 1$. The domain above the cavity is defined between $x \in[-5,10] \times y \in[-3,3] \times z \in[0,2]$, while the cavity itself is bounded by $x \in[-2.5,2.5] \times y \in[-0.5,0.5] \times z \in[-1,0]$. Steady laminar incompressible flow at $R e=500$ is obtained by imposing periodic boundary conditions along both of the $x$ - and $y$-spatial directions. In the far-field, here defined as $z \in[2,7]$, flow is driven by a constant velocity component along the $x$-spatial direction. The quality of the base flow is ensured by monitoring the residual of the solution at arbitrary locations in the domain. Figure 1 (b) shows one such result in the middle of the cavity; the time-dependence of the residual is identical for all three velocity components, is mesh-independent beyond the resolution utilized and, most importantly, following theoretical arguments on the relationship between residuals and leading global flow eigenmodes ${ }^{30}$ the slope of the logarithmic derivative curve shown is identical within the first four significant digits with the damping rate of the leading three-dimensional eigenmode, the latter to be discussed in detail in the next section.

Stability analyses using this algorithm have been performed in the cubic lid-driven cavity, as well as in the open cavity. From a numerical point of view, memory and serial CPU time scalings obtained satisfy the 
following relations

$$
\operatorname{Mem}_{T S} \sim N^{3} \quad \text { and } \quad \mathrm{T}_{T S} \sim N^{4}
$$

where $N^{3}$ is the total number of degrees of freedom (DOF) utilized to discretize the problem at hand. This implies linear scaling of memory with number of DOF and slightly above linear scaling of CPU time, as opposed to the power laws obtained when using the matrix-forming approach. ${ }^{28,29}$ This permits employing the time-stepping algorithm to analyze global instability of flows in arbitrarily complex geometries; an example of modest complexity is discussed in what follows.

\section{Applications}

Instability analyses demonstrating the performance of both the matrix-forming and the time-stepping algorithm in three configurations of increasing geometrical complexity is discussed next. We monitored stability of flow in the spanwise periodic lid-driven cavity, the three-dimensional cubic lid-driven cavity and the three-dimensional, aspect ratio $5: 1: 1$, open cavity.

\section{IV.A. Spanwise homogeneous square lid-driven cavity flow}

Flow set up in a two-dimensional square domain in which three walls are fixed and the fourth moves at a constant speed has been for decades a test bed for numerical solutions of the two-dimensional incompressible equations of motion. The realization has now set in that the spanwise homogeneous three-dimensional counterpart of this flow becomes unstable to linear modal global instability, which manifests itself through a sequence of four well-separated eigenmodes, the first being stationary and the three subsequent being traveling disturbances. These eigenmodes become exponentially unstable when the linear critical conditions, ${ }^{31}$ $\left(R e_{\mathrm{cr}}=782.61, \beta_{\mathrm{cr}}=15.37\right.$ ), are exceeded, $\beta=2 \pi / L_{z}$ denoting a periodicity wavenumber along the (homogeneous) spanwise spatial direction, z. A clear separation exists between the frequencies of the traveling modes, each of which remains approximately constant over a relatively wide band of unstable Reynolds numbers. Excellent agreement between the theoretical results for the frequency of the leading traveling disturbance $^{31}$ and earlier experimental results on the frequency of the leading perturbation of the flow ${ }^{32}$ have clearly identified the modal global linear instability scenario briefly described here as the mechanism responsible for the observed three-dimensionalization and unsteadiness in this flow.

The modal global linear instability scenario has been found to lead steady laminar two-dimensional flow into transition in lid-driven cavity flows of different cross-sectional profiles, such as the triangular cavities discussed by González et al., ${ }^{33}$ as well as in the open cavity flows studied by Bres and Colonius ${ }^{34}$ in the compressible regime. From a physical point of view, this situation is distinct from linear instability in bluffbody flows, such as the wake of the circular cylinder ${ }^{35}$ and the cascade of low-pressure turbine blades, ${ }^{36,37}$ in both of which non-modal linear mechanisms have been found to play a significant role in destabilizing the nominally steady laminar two-dimensional flow. Linear control in the prototype cavity flow is thus expected to be representative of a wider class of flows, in which well-separated linear global eigenmodes drive the laminar-turbulent transition process.

Figure 2 presents BiGlobal linear instability results obtained by application of the matrix-forming algorithm at $R e=1000, \beta=2 \pi / L_{z}=15 .^{31}$ Using the spectral collocation Chebyshev-Gauss-Lobatto (CGL) result as reference, the relative error in the amplification rate of the leading unstable stationary threedimensional eigenmode is quantified, as obtained by matrix formation and LU-decomposition, with spatial discretization based on dense and sparse spectral collocation methods based on the CGL grid, FD-q methods of $O(8)$ and $O(16)$, as well as the better known Padé, Dispersion Relation Preserving (DRP), SummationBy-Parts (SBP) and standard (STD) high-order finite-differences, all of $O(8)$.

Besides outperforming all other finite-difference methods, the most interesting result of an FD-q method in combination with the matrix forming algorithm is that three significant digits of the leading amplification rate are obtained using $N=62 \mathrm{FD}$-q 8 as opposed to $N=33 \mathrm{CGL}$ nodes discretizing each spatial direction, while if four significant digits of this quantity are required, 78 FD-q8 nodes (as opposed to $N=48 \mathrm{CGL}$ ) nodes suffice. In other words, at a cost of roughly doubling the number of discretization nodes in each spatial direction, results of equivalent accuracy with those of the (dense) spectral collocation method may be obtained using a (sparse) finite-difference stencil of bandwidth $q \ll N$. The savings in memory and CPU time for the serial LU decomposition of the matrix on a standard desktop computer are shown in Table 1 , where reductions of one order of magnitude in memory and two orders of magnitude in the CPU time 
necessary for the LU decomposition of the matrix are documented. These and other results not shown here, obtained in closed and open configurations, have led to the scalings shown in equation (11).

\section{IV.B. Cubic lid-driven cavity flow}

The substantial efficiency improvement over spectral collocation offered by the FD-q finite-difference discretization method in combination with sparse matrix storage and inversion permits solving the canonical TriGlobal linear modal instability problem in the cubic lid-driven cavity ${ }^{38,39}$ serially, on a workstation having 8GB of memory, using the matrix-forming approach. The (strongly stable) direct eigenspectrum and its associated adjoint, solutions of $(8)$ and $(9)$, respectively, at $R e=200$ are shown in Figure 3. While details of the physics of this problem are discussed elsewhere, ${ }^{40}$ Table 2 shows the computing requirements for the solution of this problem using the matrix-forming approach and the comparison of the equivalent computing cost of the time-stepping algorithm. Results of this table demonstrate the limits of the matrix forming methodology: the $O\left(N^{2}\right)$ and $O\left(N^{3}\right)$ scalings of memory and CPU time needed by the matrixforming approach, $N$ being the total number of degrees of freedom used to discretize the linearized operator, become the limiting factor as resolution needs to be increased. On the other hand, the linear scaling of the time-stepping algorithm permits recovery of the leading unstable eigenmode of this flow, recently computed for the first time independently by Giannetti et al. ${ }^{38}$ and Feldman and Gelfgat, ${ }^{39}$ also using a standard desktop. The vector of amplitude functions of velocity perturbations, $\hat{\mathbf{q}}^{\prime}=\left(\hat{\mathbf{u}}^{\prime}, \hat{\mathbf{v}}^{\prime}, \hat{\mathbf{w}}^{\prime}\right)^{T}(x, y, z)$, is shown in Figure 4 at $R e=2000$.

\section{IV.C. Aspect ratio 5:1:1 open cavity flow}

Having identified the limits of serial performance of the matrix-forming algorithm, attention is finally turned to the three-dimensional open cavity flow, using the time-stepping approach for the recovery of its global eigenspectrum. Flow in a wide range of speeds around the three-dimensional open cavity configuration is relevant to store separation and, as such, has motivated extensive theoretical investigations over decades, focusing on both hydrodynamic and aeroacoustic aspects. Invariably, simplifying assumptions needed to be made, in tune with the theoretical/numerical capabilities of the respective era. Local linear theory based on variants of the Orr-Sommerfeld equation and its compressible counterpart, the Parabolized Stability Equations and BiGlobal linear theory have all been applied in order to understand flow interactions in this problem. The assumptions underlying these theories are spatial homogeneity along two (the streamwise and spanwise) or one (the spanwise) spatial directions. Here, TriGlobal linear theory is employed for the first time to address linear instability of the inhomogeneous three-dimensional incompressible flow at a low Reynolds number, $R e=500$, at which steady laminar flow can be obtained in the configuration discussed in $\S$ III.B.

Figure 5 presents the amplitude functions of the leading stationary and the leading traveling eigenmodes of the flow. The localized nature of the amplitude functions of the leading stationary eigenmode, centered in the neighborhood of the cavity, point to the fact that the cavity acts as the origin of linear perturbations, rather than as their amplifier. On the other hand, the spatial structure of the amplitude functions of the traveling mode clearly demonstrates that events inside the cavity couple to large-scale wave-like structures traveling over the cavity; the alternative interpretation of the traveling eigenmode results, namely that the cavity modifies existing waves which travel outside it, is rather to be dismissed by reference to both the $y-$ (spanwise) and the $z-$ (wall-normal) disturbance velocity components, which dominate over the $\hat{u}(x, y, z)$ velocity component, and have both the cavity as their origin. The fact that linear perturbations exist that are localized inside the cavity is further underlined by the disturbance field streamlines shown in Figure 6 , which show activity in the two large recirculation regions formed inside the open cavity, while flow outside it remains (in this linear context) unaffected.

Interesting as it may be in its own right, the present analysis at low Reynolds number and incompressible flow is only a feasibility study as far as the store separation problem is concerned; work to complete the incompressible analysis and to extend it at higher Reynolds numbers in compressible flow is currently underway. 


\section{Conclusions}

The present contribution closes one of the open issues regarding flow control of complex flows: the efficient recovery of eigenspectra of fully three-dimensional flows. For this purpose, two different consistent methodologies have been introduced and validated in this work. First, a matrix-forming approach based on novel high-order finite different is presented, whose sparsity pattern allows the inversion of the Jacobian matrix in an very efficient way. Second, a matrix-free methodology coupling a state-of-the-art eigenspectrum recovery algorithm with a standard open source solver is presented and compared with the matrix-forming approach. TriGlobal linear instability in closed and open three-dimensional incompressible flows has been studied using two cases: a cubic lid-driven cavity and a long aspect ratio open cavity embedded into a flat plate. The adjoint eigenspectrum in the former and the direct eigenspectrum in the latter configuration are recovered for the first time. The generation of linear instabilities inside the open cavity and their connection with the flowfield outside it has been demonstrated in incompressible flow over a three-dimensional, lateralwall-bounded, aspect-ratio 5:1:1 open cavity at low Reynolds numbers. Further work is required to analyze compressible flows, where acoustic modes can be a dominant physical phenomenon. The issue of linear instability analysis of turbulent flow in high-Reynolds number flow over complex geometries remains a major open question, resolution of which may be facilitated by the algorithms presented herein. Owing to their efficiency, the same algorithms are expected to contribute to devising flow control methodologies in configurations of practical interest.

\section{Acknowledgments}

Effort sponsored by the Air Force Office of Scientific Research, Air Force Material Command, USAF, under Grant number FA8655-12-1-2004. The U.S. Government is authorized to reproduce and distribute reprints for Governmental purpose notwithstanding any copyright notation thereon.

\section{References}

\footnotetext{
272 .

${ }^{1}$ Cattafesta, L. N. and Sheplak, M., "Actuators for active flow control," Annu. Rev. Fluid Mech., Vol. 43, 2011, pp. 247-

${ }^{2}$ Bewley, T. R., "Flow Control: New Challenges for a New Renaissance," Progress in Aerospace Sciences, Vol. 37, 2001, pp. 21-58. 417.

${ }^{3}$ Kim, J. and Bewley, T. R., "A linear systems approach to flow control," Annu. Rev. Fluid Mech., Vol. 39, 2007, pp. 383-

${ }^{4}$ Collis, S. S., Joslin, R. D., Seifert, A., and Theofilis, V., "Issues in active flow control: theory, control, simulation and experiment," Prog. Aero. Sciences, Vol. 40, No. 4-5, 2004, pp. 237-289.

${ }^{5}$ Theofilis, V., "Global linear instability," Annu. Rev. Fluid Mech., Vol. 43, 2011, pp. 319-352.

${ }^{6}$ Hill, D. C., "A theoretical approach for the restabilization of wakes," AIAA Paper 92-0067, 1992.

${ }^{7}$ Giannetti, F. and Luchini, P., "Structural sensitivity of the first instability of the cylinder wake," J. Fluid Mech., Vol. 581, 2007 , pp. $167-197$.

${ }^{8}$ Ákervik, E., Hœpffner, J., Ehrenstein, U., and Henningson, D. S., "Optimal growth, model reduction and control in a separated boundary-layer flow using global modes," J. Fluid Mech., Vol. 579, 2007, pp. 305-314.

${ }^{9}$ Noack, B. R., Afanasiev, K., Morzynski, M., Tadmor, G., and Thiele, F., "A hierarchy of low-dimensional models for the transient and post-transient cylinder wake," J. Fluid Mech., Vol. 497, 2003, pp. 335-363.

${ }^{10}$ Rowley, C. W., "Model reduction for fluids using balanced proper orthogonal decomposition," Int. J. Bifurc. Chaos, Vol. 15, No. 3, 2005, pp. 997-1013.

${ }^{11}$ Gaster, M., Kit, E., and Wygnanski, I., "Large-scale structures in a forced turbulent mixing layer," J. Fluid Mech., Vol. 150, 1985, pp. 23-39.

${ }^{12}$ Pérez, J. M., Gómez, F., Blackburn, H. M., and Theofilis, V., "A shift-invert strategy for global flow instability analysis using matrix-free methods," $42^{\text {nd }}$ aiaa Fluid Dynamics Conference and Exhibit, New Orleans, LA, June 25-28 2012, AIAA Paper 2012-3276, 2012.

${ }^{13}$ Theofilis, V., "Advances in global linear instability of nonparallel and three-dimensional flows," Prog. Aero. Sciences, Vol. 39 (4), 2003, pp. 249-315.

${ }^{14}$ Saad, Y., Iterative methods for sparse linear systems, PWS Publishing, 1996.

${ }^{15}$ Chiba, S., "Global Stability Analysis of Incompressible Viscous Flow," J. Japan Soc. Comp. Fluid Dyn., Vol. 7, No. 1, 1998, pp. 20-48 (in Japanese).

${ }^{16}$ Chiba, S., "Three-dimensional global stability analysis for the time-periodic cylinder wake," Theor. Appl. Mech. Japan, Vol. 50, 2001, pp. 321-326.

${ }^{17}$ Knoll, D. A. and Keyes, D. E., "Jacobian-free Newton-Krylov methods: a survey of approaches and applications," $J$. Comp. Phys., Vol. 193, 2004, pp. 357-397.
} 
${ }^{18}$ Arnoldi, W. E., "The principle of minimized iterations in the solution of the matrix eigenvalue problem," Quart. Appl. Math., Vol. 9, 1951, pp. 17-29.

${ }^{19}$ Keller, H. B., "Numerical solution of bifurcation and nonlinear eigenvalue problems," Applications of Bifurcation Theory, edited by P. Rabinowitz, Academic Press, 1977, pp. 359-384.

${ }^{20}$ Bagheri, S., Schlatter, P., Schmid, P. J., and Henningson, D. S., "Global stability of a jet in crossflow," J. Fluid Mech., Vol. 624,2009 , pp. $33-44$.

${ }^{21}$ Tezuka, A. and Suzuki, K., "Three-Dimensional Global Linear Stability Analysis of Flow Around a Spheroid," $A I A A J .$, Vol. 44, No. 8, 2006, pp. 1697-1708.

${ }^{22}$ Gómez, F., Gómez, R., and Theofilis, V., "Coupling time-stepping numerical methods and standard aerodynamics codes for instability analysis of flows in complex geometries," $6^{\text {th }}$ Theoretical Fluid Mechanics Conference, Honolulu, HI, June 27-30, 2011, AIAA Paper 2011-3753, 2011.

${ }^{23}$ Barkley, D., Blackburn, H. M., and Sherwin, S. J., "Direct optimal growth analysis for timesteppers," Int. J. Numer. Meth. Fluids, Vol. 57, 2008, pp. $1435-1458$.

${ }^{24}$ Bagheri, S., Brandt, L., and Henningson, D. S., "Input-output analysis, model reduction and control design of the flat-plate boundary layer," J. Fluid Mech., Vol. 620, 2009, pp. 263-298.

${ }^{25}$ Luchtenburg, D. M., Günther, B., Noack, B., King, R., and Tadmor, G., "A generalized mean-field model of the natural and high-frequency actuated flow around a high-lift configuration," J. Fluid Mech., Vol. 623, 2009, pp. 283-316.

${ }^{26}$ Hermanns, M. and Hernández, J. A., "Stable high-order finite-difference methods based on non-uniform grid point distributions," Int. J. Numer. Meth. Fluids, Vol. 56, No. 3, 2008, pp. 233-255.

${ }^{27}$ Paredes, P., Theofilis, V., Rodríguez, D., and Tendero, J. A., "The PSE-3D instability analysis methodology for flows depending strongly on two and weakly on the third spatial dimension," $6^{\text {th }}$ Theoretical Fluid Mechanics Conference, Honolulu, HI, June 27-30, 2011, AIAA Paper 2011-3752, 2011.

${ }^{28}$ Gómez, F., Le Clainche, S., Paredes, P., Hermanns, M., and Theofilis, V., "Global linear instability at the dawn of its 4th decade: Recent progress and remaining challenges," AIAA J., (accepted for publication), 2012.

${ }^{29}$ Paredes, P., Hermanns, M., Le Clainche, S., and Theofilis, V., "Linear instability analysis using matrix formation and high-order finite-difference spatial discretization," Comput. Meth. Appl. Mech. Eng., Vol. (submitted), 2012.

${ }^{30}$ Theofilis, V., "On steady-state flow solutions and their nonparallel global linear instability," 8th European Turbulence Conference, Barcelona, Spain, June 27-30, 2000, Advances in Turbulencs VIII, pp. 35-38, 2000.

${ }^{31}$ Theofilis, V., "Globally-unstable flows in open cavities," AIAA Paper 2000-1965, 2000, p. 12 pp.

${ }^{32}$ Benson, J. D. and Aidun, C. K., "Transition to unsteady nonperiodic state in a through-flow lid-driven cavity," Phys. Fluids A, Vol. 4, 1992, pp. 2316-2319.

${ }^{33}$ González, L., Ahmed, M., Kühnen, J., Kuhlmann, H., and Theofilis, V., "Three-dimensional flow instability in a lid-driven isosceles triangular cavity," J. Fluid Mech., Vol. 675, 2011, pp. 369-396.

${ }^{34}$ Bres, G. A. and Colonius, T., "Three-dimensional instabilities in compressible flow over open cavities," J. Fluid Mech., Vol. 599, 2008, pp. 309-339.

${ }^{35}$ Abdessemed, N., Sharma, A. S., Sherwin, S. J., and Theofilis, V., "Transient growth analysis of the flow past a circular cylinder," Phys. Fluids, Vol. 21, 2009, pp. 044103.

${ }^{36}$ Abdessemed, N., Sherwin, S. J., and Theofilis, V., "Linear instability analysis of low pressure turbine flows," J. Fluid Mech., Vol. 628, 2009, pp. 57-83.

${ }^{37}$ Sharma, A. S., Abdessemed, N., Sherwin, S. J., and Theofilis, V., "Transient growth mechanisms of low Reynolds number flow over a low-pressure turbine blade," Theor. Comput. Fluid Dyn., Vol. 25, 2011, pp. 19 - 30.

${ }^{38}$ Giannetti, F., Luchini, P., and Marino, L., "Linear stability analysis of three-dimensional lid-driven cavity flow. Atti del XIX Congresso AIMETA di Meccanica Teorica e Applicata," (Aras Edizioni, Ancona, Italy), Ancona 14-17 Sep. 2009, 2009, pp. $738.1-10$.

${ }^{39}$ Feldman, Y. and Gelfgat, A., "Oscillatory instability in a 3D lid-driven flow in a cube," Phys. Fluids, Vol. 22, 2010, pp. 093602.

${ }^{40}$ Gómez, F., Paredes, P., Gómez, R., and Theofilis, V., "Global stability of cubic and large aspect ratio three-dimensional lid-driven cavities," $42^{\text {nd }}$ aiaa Fluid Dynamics Conference and Exhibit, New Orleans, LA, June 25-28 2012, AIAA Paper 2012-3274, 2012. 
Table 1. Memory and elapsed CPU time for the serial LU decomposition (sec) of the A matrix using dense linear algebra for CGL and sparse for CGL, FD-q16 and FD-q8.

\begin{tabular}{r|rrrr|rrrr}
\hline & \multicolumn{4}{|c|}{ Memory (MB) } & \multicolumn{4}{c}{ CPU time (sec) } \\
\hline \multirow{2}{*}{$N_{x} \times N_{y}$} & CGL $_{\text {dense }}$ & CGL $_{\text {sparse }}$ & FD-q16 & FD-q8 & CGL $_{\text {dense }}$ & CGL $_{\text {sparse }}$ & FD-q16 & FD-q8 \\
& & & & & & & & \\
\hline $20 \times 20$ & 80 & 30 & 41 & 17 & 2.3 & 0.7 & 0.8 & 0.3 \\
$40 \times 40$ & 760 & 584 & 246 & 107 & 152.7 & 34.7 & 7.6 & 2.0 \\
$60 \times 60$ & 3494 & 3078 & 775 & 284 & 1603.4 & 292.0 & 35.2 & 6.3 \\
\hline
\end{tabular}

Table 2. TriGlobal instability analysis of the regularized cubic lid-driven cavity flow in the domain $\Omega=\{x \in[0,1]\} \times\{y \in$ $[0,1]\} \times\{z \in[0,1]\}$ at $R e=200$ using matrix formation and time-stepping. In the former, FD-q finite-differences of orders $q$ and $N$ number of points in each of the three spatial directions are used. In the latter a novel variant of the Chiba algorithm, coupled with OpenFOAM ${ }^{\mathbb{B}}$ is used. The leading eigenvalue is shown in terms of damping rate $\omega_{r}$ and frequency $\omega_{i}$. In the matrix-forming method ${ }^{\dagger}$ refers to Out-of-Core while rest are In-Core calculations. The time-stepping data refer to resolutions equivalent of those used in the FD-q method.

\begin{tabular}{|c|c|c|c|c|c|c|c|c|c|c|}
\hline \multirow[b]{2}{*}{$\omega_{r}$} & \multirow[b]{2}{*}{$\omega_{i}$} & \multirow[b]{2}{*}{$N^{3}$} & \multicolumn{3}{|c|}{ Matrix-formation } & \multirow[b]{2}{*}{$\mathrm{T}_{\mathrm{AR}}(\mathrm{s})$} & \multirow[b]{2}{*}{$N^{3}$} & \multicolumn{2}{|r|}{ Time-stepping } & \multirow[b]{2}{*}{$\mathrm{T}_{\mathrm{AR}}(\mathrm{s})$} \\
\hline & & & $q$ & Memory (MB) & $\mathrm{T}_{\mathrm{LU}}(\mathrm{s})$ & & & $q$ & Memory (MB) & \\
\hline 0.41796 & 0.15894 & $20^{3}$ & 4 & 665 & 17 & 0.1 & $40^{3}$ & 2 & 64.0 & 11.9 \\
\hline 0.41475 & 0.13689 & $26^{3}$ & 4 & 2172 & 84 & 0.4 & $52^{3}$ & 2 & 128.7 & 33.4 \\
\hline 0.41601 & 0.13405 & $32^{3}$ & 4 & 5904 & 332 & 1.1 & $64^{3}$ & 2 & 229.7 & 88.4 \\
\hline 0.41585 & 0.13278 & $38^{3}$ & 4 & $3742^{\dagger}$ & $1126^{\dagger}$ & $12.4^{\dagger}$ & $76^{3}$ & 2 & 376.1 & 148.8 \\
\hline 0.41262 & 0.13152 & $20^{3}$ & 6 & 1224 & 67 & 0.2 & $60^{3}$ & 2 & 191.5 & 58.5 \\
\hline 0.41606 & 0.13191 & $26^{3}$ & 6 & 4296 & 265 & 0.7 & $78^{3}$ & 2 & 405.5 & 159.2 \\
\hline 0.41601 & 0.13191 & $32^{3}$ & 6 & $4217^{\dagger}$ & $1214^{\dagger}$ & $10.7^{\dagger}$ & $96^{3}$ & 2 & 743.4 & 451.8 \\
\hline 0.41711 & 0.13223 & $20^{3}$ & 8 & 1926 & 142 & 0.3 & $80^{3}$ & 2 & 436.0 & 180.2 \\
\hline 0.41650 & 0.13187 & $26^{3}$ & 8 & 6666 & 677 & 1.1 & $104^{3}$ & 2 & 940.9 & 512.5 \\
\hline
\end{tabular}

10 of 13 


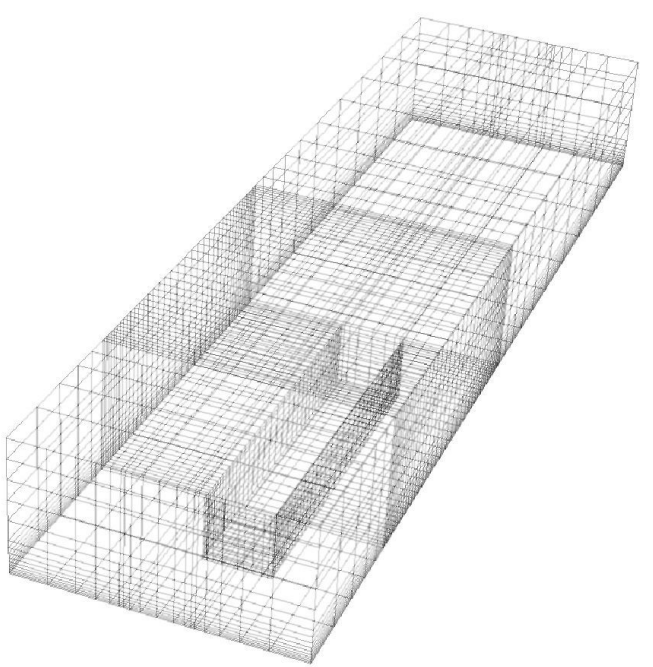

(a)

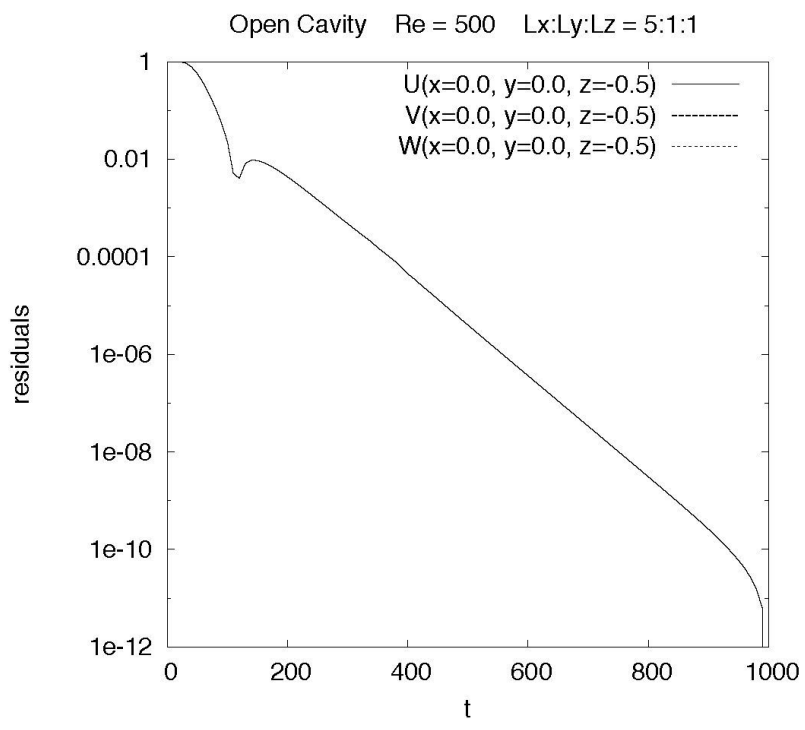

(b)

Figure 1. (a): Structured mesh around a three-dimensional open cavity. (b): Residuals of the steady laminar numerical solution in the middle of the cavity at $R e=500$

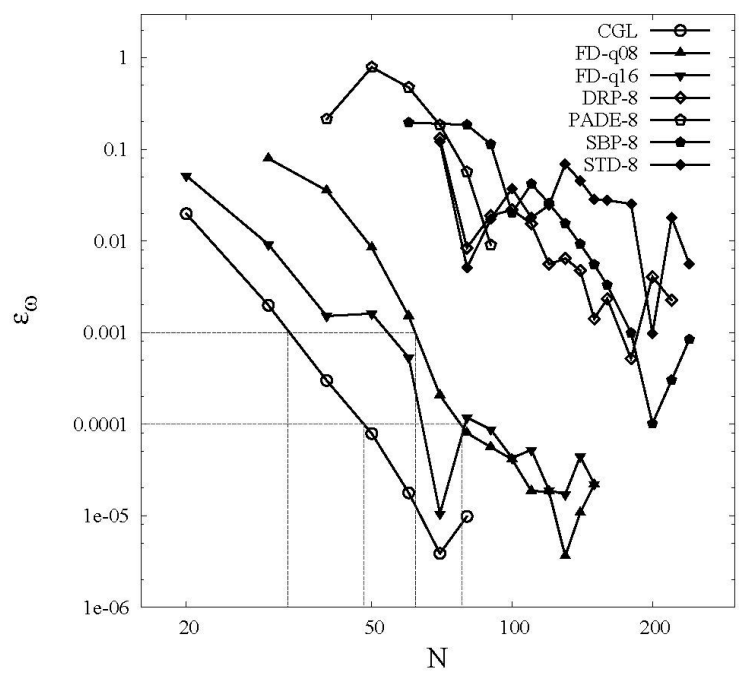

Figure 2. Convergence of the leading three-dimensional unstable stationary eigenmode in the spanwise-periodic regularized square lid-driven cavity at $R e=1000, \beta=15 .{ }^{31}$ Dashed colored lines indicate the level of discretization necessary to achieve a given relative error using CGL and FD-q8 spatial discretization. 


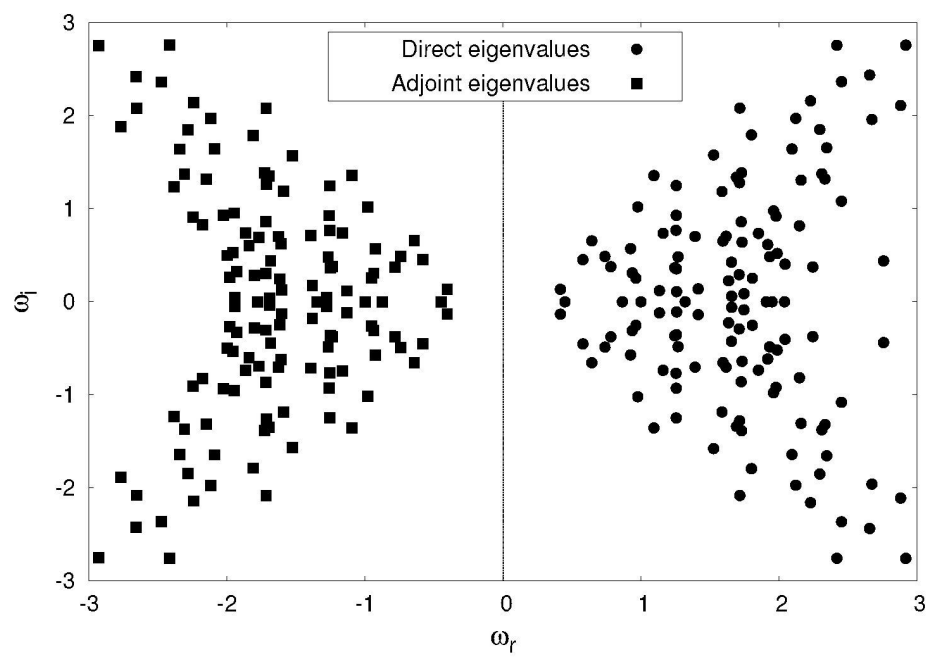

Figure 3. Direct and adjoint eigenspectra of the cubic lid-driven cavity at $R e=200$, obtained by application of the matrix-forming eigenvalue problem solver

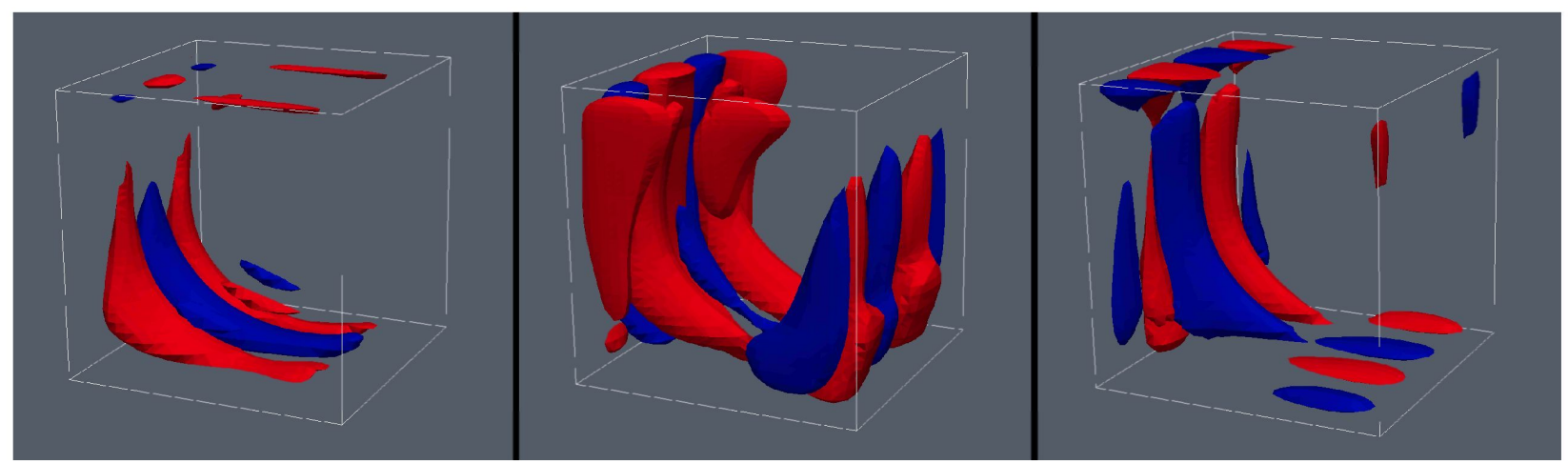

Figure 4. Leading eigenmode of the three-dimensional lid-driven cavity at $R e=2000^{38,39}$ 


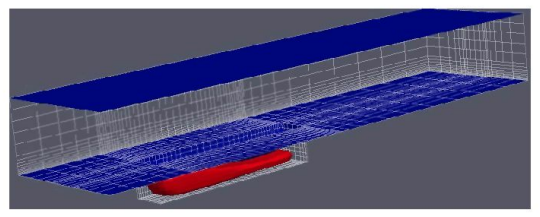

(a) $\mathrm{S} 1-\hat{u}(\mathrm{x}, \mathrm{y}, \mathrm{z})$

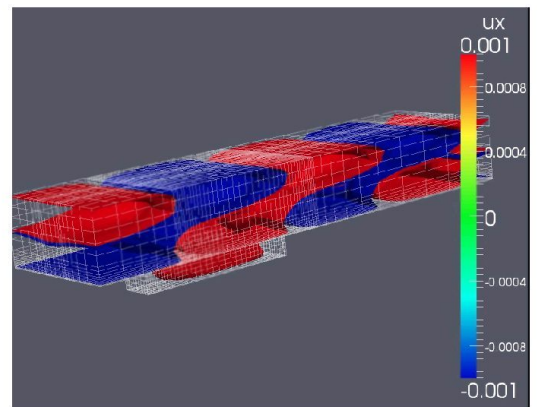

(d) $\mathrm{T} 1-\hat{u}(\mathrm{x}, \mathrm{y}, \mathrm{z})$

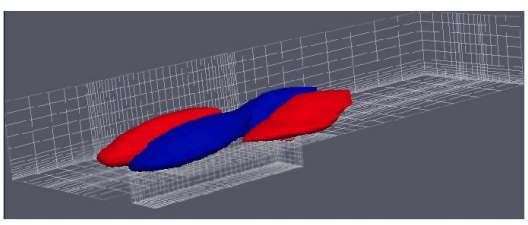

(b) $\mathrm{S} 1-\hat{v}(\mathrm{x}, \mathrm{y}, \mathrm{z})$

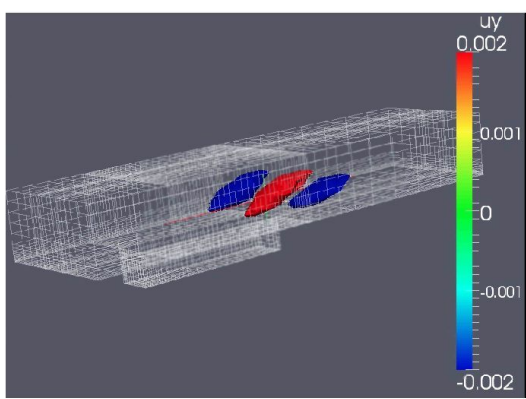

(e) $\mathrm{T} 1-\hat{v}(\mathrm{x}, \mathrm{y}, \mathrm{z})$

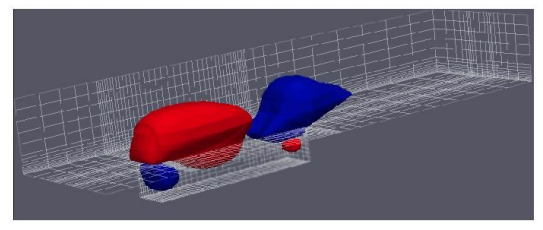

(c) $\mathrm{S} 1-\hat{w}(\mathrm{x}, \mathrm{y}, \mathrm{z})$

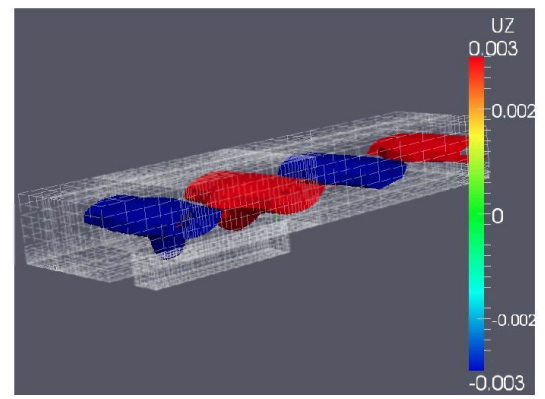

(f) $\mathrm{T} 1-\hat{w}(\mathrm{x}, \mathrm{y}, \mathrm{z})$

Figure 5. Upper row: Spatial distribution of the leading stationary (TriGlobal) eigenmode S1, of the three-dimensional aspect ratio 5:1:1 open cavity flow. Lower row: Spatial distribution of the leading traveling eigenmode $\mathrm{T} 1$.

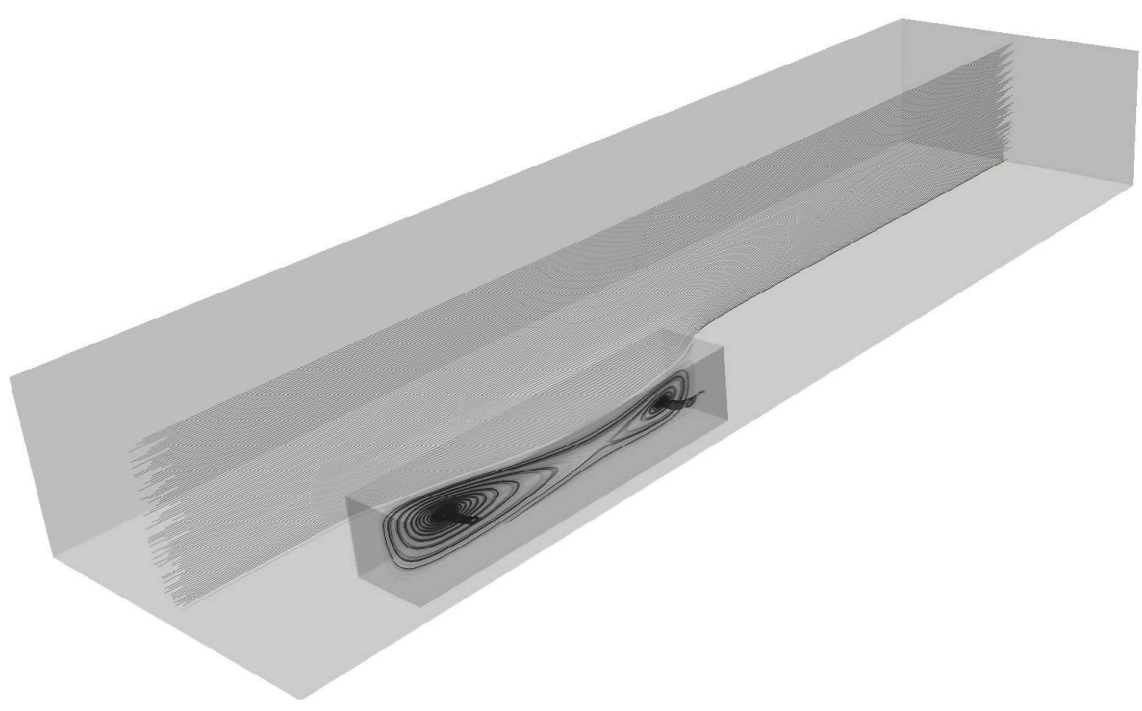

(a) $\mathrm{S} 3$

Figure 6. Mid-plane $y=0$ streamlines of the disturbance field corresponding to the third stationary eigenmode, S3, of the 5:1:1 open cavity flow at $R e=500$ 